\title{
Zur Aktualität kritisch-theoretischen Denkens für die neue Lehramtsausbildung in Österreich: Ein diversitätssensibler Entwurf inklusiver Migrationspädagogik
}

\begin{abstract}
Manfred Oberlechner*
Zusammenfassung

Pierre Bourdieus Konzeption von Bildung bzw. sein Konzept einer „reflexiven Soziologie“, Paul Mecherils disziplinäre Definition von Migrationspädagogik sowie theoretische Positionen zum Thema Inklusion und Intersektionalität bilden die theoretischen Grundlagen einer Argumentationslinie, welche notwendige Anforderungen für eine inklusive und intersektionale Migrationspädagogik als ein wesentliches Element der österreichischen LehrerInnenbildung definiert. Diesbezüglich werden demands für den Bereich Migrationspädagogik definiert betreffend curricularer Inhalte, hochschuldidaktischer Prozesse, die Personenebene und Hochschulorganisation als Ganzes. Darüber hinausgehend erfolgen die Weiterentwicklung der Migrationspädagogik mit Blick auf Inklusions- und Intersektionalitätskonzepte sowie Überlegungen zur Reflexivität in der LehrerInnenbildung bzw. zu Pädagogischer Professionalität und Reflexiver Pädagogik, die an die Kritische Theorie anschließen.
\end{abstract}

Schlagwörter: Österreichische Lehramtsausbildung, Migrationspädagogik, Inklusion, Intersektionalität, Diversität, Reflexiver Habitus, Kritische Theorie

\section{On the Relevance of critical-theoretical thinking for the new system of teacher education and training in Austria: A diversity-sensitive outline of inclusive pedagogy of migration}

\section{Abstract}

Pierre Bourdieu's concept of education and his concept of "reflexive sociology", Paul Mecheril's disciplinary definition of 'migration pedagogy' as well as theoretical perspectives on the issues of inclusion and intersectionality lay the theoretical foundations for a line of argument, which defines the necessary conditions for an inclusive and intersectional pedagogy of migration as a key element of Austrian teacher education and training. Regarding the latter, this article defines demands for migration pedagogy regarding curricular contents, didactical processes in higher education, the personal level and the overall organization of universities. Moreover, migration pedagogy is developed further with a focus on concepts of inclusion and intersectionality and additional considerations on reflexivity in teacher education and pedagogic professions more broadly, linking it to critical theory.

Keywords: Austrian teacher education and training, pedagogy of migration, inclusion, intersectionality, diversity, reflexive habitus, critical theory

\footnotetext{
*Manfred Oberlechner, Pädagogische Hochschule Salzburg Stefan Zweig. E-Mail: Manfred.Oberlechner@phsalzburg.at. Begriff und Konzept der Inklusiven Migrationspädagogik entwickelte der Autor ausführlich in: Oberlechner et al. (2017), Bramberger et al. (2017) und Kronberger et al. (2016).
} 
Lernen Sie es, die gedankliche Welt, die sich Ihnen öfnet, nicht als eine Summe von Daten anzusehen, sondern als einen Prozeß, in dem Material und lebendige Reflexion sich durchdringen und den Sie unablässig selbständig nachvollziehen müssen. Ich meine damit, daß Sie der Ihnen gebotenen Sache zugleich sich überlassen und Ihre eigene autonome Vernunft ins Spiel bringen sollen. Dieses Verhalten im Lernen, in dem Liebe und Besonnenheit sich vereinigen, kommt unmittelbar jener Elastizität des Geistes zugute, die es heute zu erneuern gilt (Horkheimer 1985c: 424).

\section{Einleitung}

Der sich seit den 1990er-Jahren vollziehende Aufstieg neuer "Populismen“ in westlichen Demokratien fordert gleichsam die Sphäre von Schulen wie auch von pädagogischen Hochschulen und Universitäten. Sie alle sehen sich veranlasst, nicht nur die eigene historischphilosophische Perspektive auf Populismus als eine populäre Entrüstungs- und Protestbewegung innerhalb eines demokratischen Systems zu überdenken. Auch ihre deontologische bzw. ethisch verpflichtete Haltung gegenüber politischen Bewegungen, von welchen sie immer öfter und heftiger für ihre humanistischen, pluralistischen und diversitätsfreundlichen Bildungsideale attackiert werden, verlangt ob der Dringlichkeit jener Erscheinungen einer Neubesinnung.

Dieses Phänomen, das die demokratischen Bausteine als Produkte der westlichen Nachkriegsordnung und damit die Trägerprinzipien des modernen, öffentlichen Schul- und Hochschulwesens bedroht, welches auf Sozialemanzipation des bürgerlichen Individuums mittels Wissensaneignung wie auch Ausbildung eines kritischreflexiven Verstandes ausgerichtet ist, lässt die kultur- und gesellschaftskritischen Diagnosen Theodor W. Adornos und Max Horkheimers aktueller denn je erscheinen: Kann der pädagogische Imperativ einer „Erziehung nach Auschwitz“ (Adorno 1997a) dem modernen Schul- und Hochschulwesen, das seine Wurzeln in der historischen Aufklärung, der Modernität bzw. Postmodernität hat, beistehen, jenen politischen Bewegungen standzuhalten, die ihrerseits das eindimensional-simplifizierende Denken, die antidemokratische und diskriminierende Einmütigkeit sowie den nationalistischen Rückzug zelebrieren?

„Unter den Einsichten von Freud“, schrieb Adorno, „... scheint mir eine der tiefsten die, daß die Zivilisation ihrerseits das Antizivilisatorische hervorbringt und es zunehmend verstärkt. ... Erziehung wäre sinnvoll überhaupt nur als eine zu kritischer Selbstreflexion ..." (Adorno 1997a: 674-676). Können Bildung und Erzie- hung, die „Organe der praktischen Aufklärung“, als Hoffnungsträgerinnen dafür dienen, dass Ausgrenzung, Diskriminierung und Rassismus, ja letztlich ein „Rückfall in das Barbarische“ dereinst nicht mehr werden sein können? Bedarf es einer „radikalen Selbstaufklärung zur Mündigkeit“ angehender Pädagogen und Pädagoginnen im Rahmen der neuen Lehramtsausbildung?' Braucht es eine Reflexion der „Tabus“ seitens der angehenden Lehrkräfte, auf dass jene, die Tabus, über und durch ihre spätere berufliche Lehrpraxis nicht weiter bestehen und die „blinde Reproduktion“ des Seienden nicht fortgeschrieben werde (Adorno 1997b; Gruschka 2003; Gruschka 2004: 135)?

Ein Teil des vorliegenden Beitrags ist daher auch die Kritik am aktuellen Trend hin zur Evidenzbasierung ${ }^{2}$

12013 wurde das „Bundesrahmengesetz zur Einführung einer neuen Ausbildung für Pädagoginnen und Pädagogen" im österreichischen Nationalrat beschlossen und damit die gesetzliche Basis für das bildungspolitische Projekt der „PädagogInnenbildung NEU“ geschaffen. (siehe https:// www.bmb.gv.at/schulen/pbneu/index.html; 4.1.2018) Am 1. Oktober 2015 startete österreichweit die neue Ausbildung für den Bereich der Primarstufe, am 1. Oktober 2016 folgte die flächendeckende Umsetzung der Lehramtsstudien für die Sekundarstufe: Der Fokus dieser Lehrer- und Lehrerinnenbildungsreform liegt im größeren Rahmen auf Veränderungen des Zusammenwirkens von pädagogischen Hochschulen und Universitäten. Alle angebotenen Lehramtsstudien sind von dieser Reform erfasst und die Studierenden werden „Bologna-konform“ ausgebildet. Die neuen Bachelor- und Masterstudien sehen demgemäß eine „wissenschaftliche und berufsfeldbezogene Qualifizierung“ im Rahmen einer „kompetenzbasierten und professionsorientierten Ausbildung" in einer Studienarchitektur vor, die in den Grundzügen einheitlich gestaltet ist. Die Kooperation der Ausbildungsinstitutionen „Pädagogische Hochschulen“ und „Universitäten“ bzw. die Bildung von vier regionalen Entwicklungsverbünden („Clusters") in Österreich soll ebendiese gemeinsame, gleichwertige Ausbildung aller Lehrer und Lehrerinnen für die Sekundarstufe ermöglichen. Schon seit 2009 hat die österreichische Bundesregierung versucht, die „Professionalisierung und Attraktivierung" des Berufes des Lehrers bzw. der Lehrerin in den Mittelpunkt andauernder nationaler Reformen der gesamten Lehramtsausbildung zu stellen; siehe https://www. bmb.gv.at/schulen/pbneu/fakten/fakten.html (4.1.2018).

2 Diese Kritik aus dem erziehungswissenschaftlichen Feld stellt nicht nur die Leistungserwartungen und Leistungsversprechen der „Evidenzbasierung" infrage, sondern macht auch auf mögliche Probleme eines theoretischen und methodischen Reduktionismus aufmerksam, der mit einer vornehmlich technisch-instrumentell verstandenen Bildungsforschung verbunden sei. Und schließlich wird auch nicht selten der Vorwurf einer affirmativen Orientierung gegenüber politischen Erwartungen formuliert. Siehe u.a. ausführlich Baumert/Tillmann (2016). 
im pädagogischen Wissenschaftsbetrieb an Universitäten wie auch an pädagogischen Hochschulen in eben jener neuen Lehramtsausbildung - ausgehend von Österreich, jedoch gleichzeitig ebenso anwendbar auf andere nationale Lehramtsausbildungen, da es sich um länderübergreifende Tendenzen einer internationalen, erziehungswissenschaftlichen Scientific Community handelt. Die Kritik erfolgt vor dem sozialphilosophischen Hintergrund der Kritischen Theorie. In Bezug auf Populismus und Bildung entspricht dies der These, dass Populismus nicht „Von außen auf die Bildung einstürzt“, sondern von der Schule, Hochschule oder Universität bzw. durch die dort dominierende „positive Pädagogik“ selbst mit hervorgebracht wird: Diese Pädagogik, die nicht im dafür nötigen Maße Aufklärung ihrer selbst betreibt, erkennen zu können, welchen Beitrag sie dazu leistet, selbst (nicht) zu sein, was sie (nicht) zu sein beansprucht, unterscheidet infolge ihrer simplifizierenden Didaktik bzw. ihres „pädagogischen Indikativs" nicht ausreichend zwischen Sein- und Normenebene: „Das Zusammenwirken ist dargestellt, als wäre es so, obwohl es doch nur sein sollte“ (Gruschka 2003). Im Gegenteil setzt sie pädagogische Idealisierungen und verkürzte, vom komplexen Ist-Zustand "herunter gebrochene“ Didaktisierungen mit der Wirklichkeit gleich, ohne sich jedoch mit den realen, soziopolitischen Widersprüchen konkret auseinanderzusetzen.

\section{Struktur der vorliegenden Arbeit}

Der Begriff „Evidenzbasierung“ bezieht sich - bevor er in einem späteren Abschnitt näher beleuchtet wird - im Folgenden zunächst lediglich implizit auf die Kritik am mechanistisch-reduktionistischen Weltbild, an der „instrumentellen Vernunft“, an der Macht der Technik, ja nicht zuletzt an der - à la Pisa - „positivistischen Forschung" per se (Horkheimer 1992c; Gruschka 2003). Im Anschluss daran wird der aktuelle kritische Diskurs zur Evidenzbasierung verstärkt in den Blick genommen und explizit auf die Kritische Theorie bezogen. Adornos und Horkheimers Kultur- und Gesellschaftskritik ist nicht obsolet, sondern im Gegenteil - so die These des Autors - vielmehr hochaktuell. Mit der Aufarbeitung ausgewählter Texte der Kritischen Theorie, besonders der „Dialektik der Aufklärung“ und anderer einschlägiger Aufsätze, beschäftigt sich der Autor daher eingehender, um nämlich deren Relevanz für den Entwurf eines entsprechend kritisch gefassten, reflexiven Lehrenden-Habitus aufzuzeigen. Hiernach wird ferner der Zusammenhang von Inklusiver Migrationspädagogik, diversitätssensibler Pädagogen- und Pädagoginnenbildung sowie kritisch-reflexivem Habitus für die neue Lehramtsausbildung erläutert und herausgestrichen.

Eine all dies flankierende und hermeneutischkritische Aufarbeitung der zentralsten Arbeiten Adornos und Horkheimers zum Themenfeld „Bildung“ stützt sich wesentlich auf den anlässlich Adornos 100. Geburtstags veröffentlichten Sammelband von Andreas Gruschka und Ulrich Oevermann (2004). In diesem wird Adornos Werk aus Gegenwartsperspektive kritisch beleuchtet und auch jene Themen werden darin diskutiert, die für den vorliegenden Text in Zusammenhang mit seiner Konzeptualisierung eines „kritisch-reflexiven Lehrenden-Habitus" von Bedeutung sind. Die wichtigsten einschlägigen, theoretischen Auseinandersetzungen des Sammelbandes werden dabei in aktualisierter Form rekonstruiert bzw. „neu gedacht“. Insbesondere der darin publizierte Artikel von Gernot Böhme, der sich dem „Eingedenken der Natur im Subjekt“ bzw. der „Geburt des Subjekts aus dem Schmerz" widmet, und Andreas Gruschkas „Kritische Pädagogik nach Adorno“ offenbaren hierfür ein inspirierendes analytisches Repertoire.

Insbesondere zwei der innerhalb der Bildungswissenschaft intensiv diskutierten Texte Adornos, in welchen dessen grundlegend ambivalentes Verhältnis zur bzw. scharfe Kritik an der Pädagogik seiner Zeit zum Ausdruck kommt, d.i. „Tabus über dem Lehrberuf“ (Adorno 1997b) und „Erziehung nach Auschwitz“ (Adorno 1997a), spielen in diesem Kontext eine gewichtige Rolle, denn beide behandeln unter anderem jene Fragestellung, welcher sich der Autor mit Blick auf die neue Lehramtsausbildung in Österreich annähern wird. ${ }^{3}$

Pierre Bourdieus Habitusbegriff (Bourdieu 1983; Bourdieu/Passeron 1971) und sein Konzept einer „reflexiven Soziologie“ (Bourdieu 1995), deren Anspruch es ist, die gesellschaftlichen Bedingungen, unter denen sozialwissenschaftliche Forschung betrieben wird, systematisch zu berücksichtigen, werden daraufhin ausführlicher behandelt und vom Autor zum Entwurf eines „kritisch-reflexiven Lehrer- und Lehrerinnenhabitus“ herangezogen (siehe dazu die bildungswissenschaftlichen und bildungssoziologischen Studien u.a. von Friebertshäuser, Rieger-Ladich/Wigger 2006). Dieser Begriff des „kritisch-reflexiven Lehrer- und Lehrerinnenhabitus" wurde in der Literatur bereits vielfach aufgegriffen

3 Auf Bildungsfragen bezogene Literatur zu Adorno und Horkheimer gibt es zur Genüge, etwa aus der Kritischen Erziehungswissenschaft: siehe z.B. Armin 2001; Claußen/ Scarbath 1979. 
und innerhalb des Faches gar reichlich diskutiert, existieren hierzu doch inzwischen geradezu zahllose $\mathrm{Pu}$ blikationen - den einen Begriff von „Reflexivität“ oder „deliberate practice“ (Williamon 2009) oder auch des „reflective practitioners“ (Schön 1983) gibt es bei aller Fülle an Abhandlungen jedoch nicht, nicht zuletzt deswegen, weil die Thematik schon seit etwa vierzig Jahren zwar gründlich, aber durchaus sehr kontrovers aufgearbeitet wurde (vgl. dazu jüngst Häcker 2017). ${ }^{4}$

Im letzten Abschnitt wird schließlich das Konzept einer kritisch-reflexiven Lehrer- und Lehrerinnenbildung mit Bezug auf ein Inklusives Migrationsparadigma thematisiert: Theoretische Anknüpfungspunkte finden sich hier in der von Paul Mecheril (2004) vorgeschlagenen disziplinären Spezialisierung einer „Migrationspädagogik" sowie in den theoretischen Positionen zum Thema „Inklusion“. Hier insbesondere die internationalen (bildungs-)politischen Normsetzungen entsprechend der UNESCO-Erklärung von Salamanca (1994) samt deren normativem Appell, „Regelschulen“ mit entsprechend „inklusiver Ausrichtung“ als besten Weg zu erachten, um Diskriminierungen zu bekämpfen. Mit anderen Worten: „Inklusive Bildung“ wird hier auf die „gesamte Diversität“ - einschließlich Migration bezogen (Allemann-Ghionda 2013).

Ein umfassender Migrationsbegriff ist notwendige Grundlage dieser Inklusiven Migrationspädagogik, denn er umfasst u.a. Fragen der Ein- und Auswanderung, regulären und irregulären Migration, Arbeits- und Fluchtmigration, Umweltmigration, Konstruktion von Fremdheit, Gender und Migration, Identitätskonstruktionen, Transmigration, Exil, Braindrain, Braingain, Brainwaste - und vieles mehr, das heißt all die Migrationsphänomene, die für inklusive Bildungskontexte bildungsrelevant sind und daher auch gesehen und mitgedacht werden sollen, wenn sie auf individuelle wie gemeinschaftliche/gesellschaftliche Bildungsverläufe einwirken und somit für inklusive Bildungsinstitutionen maßgebend sind (Mecheril/ Oberlechner 2016: 155-156). Diese Migrationspädagogik als (relativ) neues Paradigma versteht sich vor dem Hintergrund einer bürger- bzw. menschenrechtlich geprägten westlichen Zivilgesellschaft als Beitrag zu einem „inklusiven Gesellschaftsverständnis“ und berührt mit ihrer Selbstverortung die Domäne ande-

4 Der Terminus der „Reflexiven Erziehungswissenschaft“ ist innerhalb des pädagogischen Diskurses ebenfalls bereits seit Beginn der 199oer-Jahre etabliert (siehe u.a. Lenzen 1991). rer Wissenschafter und Wissenschafterinnen, die den Begriff der „Inklusion“ bzw. „Integration“ schon vorher für sich und ihre Disziplin in Anspruch nahmen und auf Grund dieses chronologischen Vorsprungs einen wissenschaftstheoretischen Besitzanspruch deklarieren könnten. ${ }^{5}$ Bildung für alle als internationale Leitidee und als Prinzip der „Inklusion“ wird dementsprechend auf supranationaler Ebene propagiert, die Begriffe „Inklusion“ und „Integration“ hierbei in Dokumenten der Vereinten Nationen und UNESCO synonym verwendet (Allemann-Ghionda 2013: 126; Furch 2009: 177). „Inklusive Bildung“ ist jedenfalls auf die gesamte Diversität bezogen und nicht auf die gemeinsame Bildung von Schülerinnen und Schülern „mit oder ohne Beeinträchtigung“ verengt. Signifikant überrepräsentiert ist beispielsweise bis heute in Österreich der Anteil von Kindern „mit Migrationshintergrund“ unter Schülern und Schülerinnen mit „sonderpädagogischem Förderbedarf“. Einer Aussonderung von Schülern und Schülerinnen mit Migrationshintergrund in separate Förderschulen ist (insbesondere wenn es dabei primär um Fragen der Sprachentwicklung und -kompetenz bei Kindern mit nicht deutscher Erstsprache geht) aus dieser inklusiven Sicht entgegenzusteuern. ${ }^{6}$

5 Aus dem Blickwinkel ,integrativer“, „,interkultureller" und „feministischer Pädagogik“ thematisiert Annedore Prengel 2003 erstmals in ihrem zum Standardwerk gewordenen Buch „Pädagogik der Vielfalt“ ein Konzept, in dem sowohl unterschiedliche Geschlechterrollen, ethnische Hintergründe, religiöse Überzeugungen, soziale Lagen als auch Fähigkeiten und Beeinträchtigungen in den Blick genommen werden. Mit dem Begriff der „egalitären Differenz" theoretisiert sie darauf aufbauend eine „inklusive Gesellschaft", in der die Respektierung der Unterschiede und die Anerkennung von Gleichheit interdependent sind (Prengel 2006: 11). Als Anregung für diesen Prozess haben Tony Booth und Mel Ainscow (2011: 28) mit dem „Index of Inclusion“ einen Wertekanon als Fundament einer "guten Bildung“ sowie einen „inklusiven Zugang“ in den Curricula gefordert.

6 Es bleibt noch abzuwarten, ob die Thematik Migration bzw. Migrationspädagogik in den "Querschnittsmaterien" der Lehramtsausbildungen in Österreich eigenständig inkludiert bleiben oder darin in einer belanglos-oberflächlichen Vielfalt „untergehen“. Denn es fehlen in Kerncurricula der neuen Lehramtsausbildung sowohl für die Primar- und Sekundarstufe die schwerpunktmäßigen Lehrveranstaltungen, die allein dem Themenfeld Migrationspädagogik in der oben genannten umfassenden Breite vorbehalten sind; und auch was den Teilfokus Mehrsprachigkeit in Bezug auf Migrationspädagogik betrifft, ist zu konstatieren, dass dieser zwar in den neuen Curricula expliziter ist, konkrete Herangehensweisen bzw. die verpflichtende Verankerung entsprechender Lehrinhalte in den verpflichtenden Kerncurricula jedoch ausbaufähig bleiben. 


\section{Kritische Theorie}

Die „Dialektik der Aufklärung“ und „Zur Kritik der instrumentellen Vernunft" kritisieren die aufgrund ihres reduktionistischen Naturbilds sich in einer "Sackgasse“ befindende Vernunft. Die dialektische Konsequenz, die sich aus dieser „Vergewaltigung“ ergibt, bringt Horkheimer auf den Punkt: Je mehr Apparate zur Naturbeherrschung erfunden sind, desto mehr müsse ihnen gedient werden (Horkheimer 1992a: 94) - und desto mehr werden Menschen dabei sich selbst entfremdet. Dabei verstrickt sich dieses Aufklärungsdenken in einen Widerspruch: „In der menschlichen Vernunft finden sich einige unausrottbare Ideen wie Freiheit, Gerechtigkeit und Menschlichkeit. Die Aufgabe dieser Ideen käme der menschlichen Vernunft gleich" (Horkheimer 1989: 357). Diese positivistische Auffassung von Wahrheit ist reduziert auf ein methodologisches Prüfverfahren, in dem „Richtigkeit“ „Wahrheit“ bedeutet. Dieser mathematische Formalismus reduziert die Wirkkräfte auf wenige „harte Fakten“, die nur noch wiederholt werden. In diesem Sinn ist Positivismus nach Horkheimer als ideologisch-affirmative Wissenschaft zu verstehen, die sich auf die bloße Wiederholung von vorgefundener Realität beschränkt (Horkheimer 1970: 21).

Dieser Faktizitätsbegriff vernachlässigt die Einsicht, dass die soziale Welt gewachsenes Produkt gesellschaftlichen Handelns ist. Die Angst, von Tatsachen abzugehen, präpariert jedoch den „verdorrenden Boden für die gierige Aufnahme von Scharlatanerie und Aberglauben" (Horkheimer/Adorno 1987: 18). Wissenschaftliche Erkenntnis degeneriert so $\mathrm{zu}$ einem Verwertungswissen, welches jeder Ideologisierung offensteht. Aus diesem Grund fordert Horkheimer die Durchdringung von Philosophie und Einzelforschung: Kombiniert soll die Kluft zwischen Tatsachenforschung und Sozialphilosophie überwunden werden.

Die auf Instrumentalität regredierte Vernunft hat sich durch Naturbeherrschung und soziale Herrschaft auch in Gestalt von „Bildung“ - zu einem quasi-mythischen Bann zusammengeschlossen. Die Abhandlung "Zur Kritik der instrumentellen Vernunft“ schildert diesen Prozess als Verdrängung einer als „objektiv“verstandenen wert- und sinnkonstituierenden Vernunft durch eine „subjektive“, instrumentell-formalistische Vernunftauffassung. Was nur noch zählt: die kalkulatorische Fähigkeit für subjektiv-nutzenmaximierendes Handeln. Enttäuscht von der Hegelschen Philosophie (sie habe das Individuum, seine Hoffnungen, Erwartungen und Sehnsüchte der „Vernünftigkeit des sozialen
Ganzen“ geopfert), fordern die kritischen Theoretiker bzw. Theoretikerinnen daher die bewusste Akzentuierung des erkenntnisleitenden Interesses: Erkenntnis bedarf des Interesses an einer Aufhebung von sozialen Entfremdungsprozessen, durch die das Individuum seine Identität $\mathrm{zu}$ verlieren droht. Beim kritischen Denken geht es daher nicht darum, formalkonsequent zu denken, sondern vielmehr eine dynamische Offenheit zu entwickeln, um Widersprüchlichkeiten ständig Raum zu geben.

\section{Kritik der Evidenzbasierung im Bildungskontext}

Wenn instrumentelle Vernunft zum Maßstab technisch-instrumentell verstandener Bildungsforschung wird und damit ein theoretischer und methodischer Reduktionismus verbunden ist, da letztlich nur noch gemessen wird, was messbar ist, steht der Vorwurf der affirmativen Unterordnung gegenüber vorgefundenen Sachzwängen im Raum: Nur diejenigen Bildungs„Tatsachen“ werden bestimmt, die einen unmittelbaren Nutzen versprechen. Aufgrund dieser Maxime, gesichertes Wissen ausschließlich aus Beobachtung und Erfahrung induzieren zu wollen (unter Ausblendung all jener sozialen Phänomene, die sich diesem Erkenntnisweg entziehen), findet hier unweigerlich eine Hinwendung zum faktisch Gegebenen statt, wenn nicht empirisch überprüfbare, nicht operationalisierbare Sinn- und Existenzfragen in Bildungs- und Erziehungsprozessen aus dem Blick geraten. Diese Kritik wird daher auch aus erziehungswissenschaftlich-kritischer Perspektive geäußert (u.a. Baumert 2007; Baumert/ Tillmann 2016; Brügelmann 2015; Gruschka 2006; Bellmann/Müller 2011): Vor allem Herzog (2016: 201) kritisiert am Paradigma der „evidenzbasierten Pädagogik“, dass es von der Wirksamkeit pädagogischer Technologien ausgeht, Kommunikation als Medium pädagogischer Wirksamkeit jedoch missachtet und demnach ein unangemessenes Bild der Handlungsprobleme von Lehrern und Lehrerinnen abgibt. Zudem geht es dieser Pädagogik nicht um „Bildung“, sondern um Leistungsmaximierung, wenn sie mit einseitigen Methoden und verkürzten Theorieansätzen nur Ergebnisse für eine technokratische Verwertung des schulischen Lernens liefert, wodurch „Bildung“ letztlich bloß eine effektivere Humankapital-Produktion bedeutet (u.a. Terhart 2002; Radtke 2015). Das wissenschaftstheoretische Credo dieser (von Horkheimer wohl als „traditionell“ klassifizierten) Bildungsforschung prolongiert den historischen Positivismus. Wenn Wissenschaftlichkeit 
und Nützlichkeit in der Bildungsforschung so direkt aneinandergekoppelt sind, ist die Abgrenzung gegenüber anderen Wissensformen, präziser: gegenüber anderen Formen pädagogischen und erziehungswissenschaftlichen Wissens, impliziert.

\section{Bourdieus Kapitalbegriff und ein kritisch- reflexiver Lehrenden-Habitus}

Bourdieu erweitert den Kapitalbegriff in seiner Bedeutung, indem er nicht nur im Ökonomischen, dem Besitz von Produktionsmitteln, Geld und Kapital („ökonomisches Kapital“), sondern auch in sozialen Beziehungen („soziales Kapital“) sowie Bildung („kulturelles Kapital“) eine Quelle von Gestaltungsmacht sieht (Bourdieu 1983: 185). Er bezeichnet mit anderen Worten den je eigenen „Stil“ des handelnden Individuums oder einzelner Gruppen, der sich aus Erfahrungen der Vergangenheit speist und auf die Gegenwart und Zukunft hinwirkt (Bourdieu/Passeron 1971: 20). Es ist daher nur konsequent, von einem „Lehrer- und Lehrerinnenhabitus“ $\mathrm{zu}$ sprechen, gemeint als „doppelte Professionalisierung“ bzw. Ausbildung eines „doppelten Habitus" in der neuen Lehramtsausbildung als „wissenschaftlich-reflexiver Habitus" und „Habitus des praktisch-routinisierten Könnens“ (Helsper 2001: 13) - bzw. dies in Verbindung mit pädagogischer Professionalität für die Inklusive Migrationspädagogik einzufordern: Als „verinnerlichtes Kulturkapital“ kann sich dieser Lehrenden-Habitus als „dauerhafte Disposition“ von Lehrpersonen präsentieren. Die Ausbildung zu einer Inklusiven Migrationspädagogik sowie die entsprechende Akkumulation von inklusivmigrationspädagogischem Fachwissen im Rahmen der Lehramtsausbildung werden somit zum „inkorporierten Kapital“:

„Die Akkumulation von Kultur in inkorporiertem

Zustand - also in der Form, die man auf französisch ,culture, auf deutsch ,Bildung; auf englisch, cultivation nennt - setzt einen Verinnerlichungsprozeß voraus, der in dem Maße wie er Unterrichts- und Lernzeit erfordert, Zeit kostet. Die Zeit muß vom Investor persönlich investiert werden"... (Bourdieu 1983: 187).

Dieses „Bildungskapital“ ist eine Unterform des inkorporierten Kulturkapitals. Wer an pädagogischen Hochschulen oder Universitäten im Rahmen seines Lehramtsstudiums daher inklusiv-migrationspädagogisch ausgebildet werden will, sollte allerdings zuvorderst an sich selbst arbeiten. Erst auf diesem Weg kann das inklusiv-migrationspädagogische Fachwissen zum „inkorporierten Kulturkapital“ von Lehrern und Lehrerinnen werden (Bourdieu 1983: 187).

\section{Anforderungen an die Inklusive Migrationspädagogik}

Lernen nach Inklusiver Migrationspädagogik braucht zuallererst fachdidaktische Forschung $\mathrm{zu}$ inklusiver, fachbezogener, migrationspädagogischer Lehre. Neben der fachdidaktischen Perspektive auf Migrationspädagogik ist daher eine inklusionspädagogische Erweiterung notwendig, wenn Migrationspädagogik zum Bestandteil einer „Inklusiven Pädagogischen Hochschule“ wird. Inklusiv-migrationspädagogisches Lernen greift außerdem nicht auf vereinfachende Identitätskonstruktionen zurück. Für den inklusiven Bildungskontext unbeachtet bleiben sonst all die individuellen Verortungen, die aus mehrfachen und gleichzeitigen Identitätsquellen schöpfen (Oberlechner 2017b: 83). Die Inklusive Migrationspädagogik benötigt vielmehr ständiges, selbstkritisches Reflektieren bzw. permanente eigene wie fremde Standortbestimmungen bezüglich der Anerkennung von Differenz und Pluralität in Hinsicht auf gesellschaftliche Diskriminierungs- und Privilegierungsstrukturen. Dann erst kann differenzsensiblen Didaktiken im Rahmen einer inklusiven Lehramtsausbildung ein tatsächlicher Stellenwert zukommen - was keinesfalls eine Anfertigung inklusiv-migrationspädagogischer Tools inklusive taxativer Rezeptaufzählungen handlungsleitender Lern- und Lehrmethoden bedeutet. Mit anderen Worten: Diese kritische (Selbst-)Reflexivität soll im oben genannten kritisch-theoretischen Sinn daher antipositivistisch sein. Sie geht davon aus, dass sich das Denken in die Realität zwar einlassen muss, und meint gleichzeitig, dass es der Distanz dazu bedarf, um selbstkritisch sein zu können.

$\mathrm{Zu}$ dieser Kritik gehört nicht nur das negative, skeptische Moment, sondern auch die innere Unabhängigkeit, dem pädagogischen Zeitgeist widerstehen zu können. Ein kritischer Lehrender bzw. eine kritische Lehrende hebt dann gesellschaftlich vermittelte Widersprüche dialektisch - im Sinn ihrer diagnostischen Feststellung - auf, ja vertieft und bewahrt sie geradezu, um sie anschließend auf ein neues Niveau zu heben, das andere Sichtweisen zulässt. Nicht das „Inklusiv-Pädagogisch-Widerspruchslose“ zu schaffen, sondern vielmehr real Widersprüchliches so zu erkennen und zu begreifen, dass es adäquat dargestellt und verändert werden kann, ist maßgeblich und zentral. 
Gerade im Spannungsfeld von Inklusiver Migrationspädagogik zur Leistungsbeurteilung und -verwertung an Pädagogischen Hochschulen und Universitäten, die Beurteilungs-, Selektions- bzw. Allokationsaufträgen nachkommen (müssen), ist die entsprechend selbstreflexive Inklusive Migrationspädagogik von gesellschaftlichen Interferenzen, Paradoxien und Ambiguitäten geprägt. Eine Reflexivität als habitualisierte bzw. institutionalisierte Form einer reflexiven, referenziellen bzw. selbstreferenziellen, d.h. rückbezüglichen bzw. selbstbezüglichen Art des Denkens (Häcker 2017: 23) ist hierfür ein essentieller Beitrag zum Umgang mit inklusiv-migrationspädagogischen Anforderungen, wenn die bildungsinstitutionellen Felder „Pädagogische Hochschule“ und „Universität“ ebenfalls eine reflexive Kultur aufweisen (Mecheril/ Oberlechner 2016). Die Frage, wie diese Bildungsorte zu „Orten der Reflexivität“ werden, zu Orten, an denen Professionelle sich gleichzeitig als Lernende begreifen, steht im Vordergrund:

„Das Pathos der Schule heute [und der pädagogischen Hochschule oder Universität - Anm.d.Verf.], ihr moralischer Ernst ist, daß inmitten des Bestehenden nur sie, wenn sie sich dessen bewußt ist, unmittelbar auf die Entbarbarisierung der Menschheit hinzuarbeiten vermag. Mit Barbarei meine ich ... das Äußerste: wahnhaftes Vorurteil, Unterdrückung, Völkermord und Folter; darüber soll kein Zweifel sein. Dagegen anzugehen, ist ... vor allem anderen an der Schule. Deshalb ist es, trotz aller theoretisch-gesellschaftlichen Gegenargumente, gesellschaftlich so eminent wichtig, daß sie ihre Aufgabe erfüllt und dazu hilft, daß sie des verhängnisvollen Erbes an Vorstellungen sich bewußt wird, das auf ihr lastet" (Adorno 1997b: 672).

Diese selbstreflexive Lehramtsausbildung verwendet einen Migrationsbegriff, der über moralische Fallstricke, weltanschauliche Gratwanderungen und Gefahren der Instrumentalisierung informiert ist. Diese reflexivinklusive Migrationspädagogik reflektiert Kulturalisierungen und Essentialisierungen, denn Studierende wie Lehrende müssen im Rahmen der neuen Lehrerund Lehrerinnenbildung ihr pädagogisches Handeln begründen können. Theoriegeleitetes Nachdenken über Leistungen und Exzellenzen an pädagogischen Hochschulen und Universitäten ist somit erforderlich. Dies verlangt Reformen bzw. den Auftrag an pädagogische Hochschulen, diesbezügliche Grundlagenforschung nicht an Universitäten „auszulagern“ und selbst nur mehr einer „berufsfeldbezogenen Forschung“ das Wort $\mathrm{zu}$ reden, die allein zulässt, „was in der Praxis beim Kind direkt ankommt“.
Denn dieser Zwang zur unmittelbaren Wirkungsmessung im Anspruch evidenzbasierter Bildungsforschung bedeutet eine ungerechtfertigte Einschränkung: Es geht vielmehr um allgemeines bildungswissenschaftliches Grundlagenwissen, das ein kritisches Bewusstsein in der Lehramtsausbildung erst ermöglicht. Es sind grundsätzliche Fragen zu stellen, etwa danach, welches Wissen von Bildungsinstitutionen selbst legitimiert und welches abgewehrt bzw. disqualifiziert wird: Welcher Wert wird welchem Wissen in Bildungsinstitutionen warum zu-oder abgesprochen? Es bedarf eines kritischreflexiven Diskurses darüber, welche personalen und sozialen Potenziale und Ressourcen Lehramtsstudierende mitbringen, die in den Leistungskulturen von pädagogischen Hochschulen und Universitäten zu wenig berücksichtigt sind.

\section{Diversitätswahrnehmung und reflexiver Lehrenden-Habitus}

Differenzlinien erzeugen Fremdheiten und damit Grenzen, gleichzeitig wiederum Diversitäten bzw. ständig andere Identitätskonstruktionen und -kombinationen. Eine Ambiguitätstoleranz als Bestandteil kritischer Reflexionskompetenz basiert auf der Einsicht, dass ein Mensch nicht alles und alle bzw. jeden und jede verstehen kann. Ein totalitärer „Verstehens-Anspruch“ von Fremdheiten, seien sie physischer oder psychischer Natur, wäre im Sinne Horkheimers und Adornos ein Mythos - blind und verlockend zugleich, letztendlich irreführend. Denn Diversität braucht Fremdheiten wesentlich. Und jene - ob nun die eigenen oder die des/r Anderen - brauchen Autonomie, Toleranz von innen heraus und sich selbst gegenüber sowie von außen her. Sie benötigen - mehr noch - Indifferenz, ja ein Existenzrecht ohne korrigierenden Eingriff. Es muss ein Recht auf Nicht-Inklusion oder Nicht-Integration persönlicher Privatheit geben.

Inklusive Diversitäts- und Migrationspädagogik, die sich der „Logik der Nützlichkeit“ unterwirft, läuft Gefahr, Vielfalt stets nach ihrem Nutzen zu bewerten. Dabei gerät man in die „Falle“, Merkmale, die keinen Nutzen abwerfen oder im ökonomischen Sinne etwas „kosten“, negativ zu bewerten. Ein theoretisch-kritisch ausgerichteter Ansatz kommt somit nicht umhin, egalitär inspirierte Ideen, wie z.B. „Gleichheit“ oder „Gerechtigkeit“, ins Zentrum des eigenen Bildungsbegriffs zu stellen, soll Diversität bzw. Migration nicht vor den „Karren“ der Verwertungslogik gespannt werden. Es ist daher nicht verwunderlich, dass meist ökono- 
misch orientierte Diversitäts-Ansätze Kategorien wie „Klasse“ entweder unter den Tisch fallen lassen oder als „unveränderlich“ bzw. „notwendig“ deklarieren. Aus Perspektive der Kritischen Theorie käme dies einem „Rede- und Denkverbot" gleich. Eine Inklusive Migrationspädagogik in Bezug auf einen reflexiven Lehrer- und Lehrerinnenhabitus stellt sich jedoch bewusst in den Dienst von „Gleichheit“, „Gerechtigkeit“ und „Freiheit“, welche zu fördern sie zuallererst trachtet. Denn was ist letzten Endes die Konsequenz einer Instrumentalisierung von Vernunft, wenn nicht ihr Entbehren ebenjener Werte? Oder, mit Horkheimers Worten:

„Gerechtigkeit, Gleichheit, Glück, Toleranz, alle die Begriffe, die ... in den vorhergehenden Jahrhunderten der Vernunft innewohnen oder von ihr sanktioniert sein sollten, haben ihre geistigen Wurzeln verloren. Sie sind noch Ziele und Zwecke, aber es gibt keine rationale Instanz, die befugt wäre, ihnen einen Wert zuzusprechen und sie mit einer objektiven Realität zusammenzubringen. ... Wer kann sagen, daß irgendeines dieser Ideale enger auf Wahrheit bezogen ist als sein Gegenteil" (Horkheimer 1992c: 32).

\section{Resümee}

Grundlagenorientierte Theorie und Kritik müssen nicht immer in praktisch-pädagogische Lösungsvorschläge münden. Zur Kritik gehört nicht nur das negative, skeptische Moment, sondern zugleich die innere Unabhängigkeit des/r Einzelnen, dem launigen Zeitgeist zu widerstehen: „Die einzig wahrhafte Kraft gegen das Prinzip von Auschwitz wäre Autonomie, wenn ich den Kantischen Ausdruck verwenden darf; die Kraft zur Reflexion, zur Selbstbestimmung, zum NichtMitmachen“ (Adorno 1997: 679). Im „Negativen“ steckt die Bejahung des anderen - „mittelbar Positiven“. Diese „negative Dialektik“ setzt sich für die selbstständige Rolle von Kritik ein: „Sie bezeichnet im Gedanken an ein Anderes die Gesellschaft, in der wir leben, als das schlecht Bestehende. Das Absolute positiv darzustellen, wäre im letzten Sinne jedoch eine Art Götzendienst" (Horkheimer 1985d: 287).

Nichtsdestotrotz kann mit der in den vorangehenden Abschnitten skizzierten Argumentationslinie eine Inklusive Migrationspädagogik als ein Element der neuen Lehramtsausbildung gefordert und gefördert werden; und gleichzeitig sind damit weitreichende Vorstellungen einer kritischen, selbstreflexiven und inklusiven Lehramtsausbildung verbunden. Diese geht von einer speziellen Wissensvermittlung aus, bei der Migrationspädagogik um Inklusive Pädagogik erwei- tert wird, um eine gerechtere Teilhabe an Hochschulbildung bei diversitätssensiblem Blick der Pädagogen und Pädagoginnen auf Differenzlinien in Bildungsprozessen zu erreichen (Oberlechner 2017a).

Notwendig hierfür ist ein breites bildungs-, diversitäts- bzw. migrationssoziologisches Wissen, das eine „Postulatepädagogik“ (Gruschka 2004: 138), bei welcher allzu rasch vom Sollen auf das Sein geschlossen wird, zu vermeiden weiß und damit ein gesellschaftskritisches Bewusstsein für die neue Lehramtsausbildung erst ermöglicht. Ein solchermaßen reflexiver Lehrerund Lehrerinnenhabitus könnte Wissen bereitstellen, welches bildungswissenschaftliches Theoretisieren bzw. pädagogisches Denken und Handeln mit Bezug auf eine Inklusive Migrationspädagogik unterstützt. Dafür unabdingbar ist, dass gesamtgesellschaftliche Fragen, die über den unmittelbaren Lern-Lehr-Kontext hinausgehen, in Forschung und Lehre Pädagogischer Hochschulen und Universitäten fix inkludiert sind: Dies kann im Fall der Inklusiven Migrationspädagogik bis hin zu sozialphilosophischen Fragestellungen reichen und sich im Speziellen an die Kritische Theorie anlehnen, um den Ideologiegehalt von Bildungstheorien aus ideologiekritischer Sicht zu hinterfragen.

Ein damit einhergehender, kritisch-theoretischer Bildungsauftrag an Pädagogische Hochschulen und Universitäten setzt an folgenden Hauptaspekten an: an der Reflexionskompetenz von Lehrenden wie Lernenden, den Merkmalen der Bildungsorganisationen sowie den dort herrschenden Lehrformen und Lehrinhalten. Die (angehenden) Lehrenden brauchen Möglichkeiten, aktuelle Migrationsthemen historisch kontextualisieren $z u$ können. Gefragt ist dafür eine gesellschaftsanalytische und geschichtsbewusste Auseinandersetzung mit Migrationsphänomenen (zum Beispiel im Rahmen postkolonialer Forschung), damit die Hintergründe dessen, was in- und außerhalb von Bildungsinstitutionen geschieht, professionell und nicht nur mittels Alltagstheorien gedeutet werden (Doğmuş, Karakaşoğlu, Mecheril 2017: 7). Dies wirft die Frage auf, inwiefern sozialer Wandel zu sozialen Differenzlinien mit Bevorzugungen oder Benachteiligungen bzw. multiplen „Anerkennungs- bzw. Missachtungserfahrungen“ der in den Bildungsprozess inkludierten Personen führt (z.B. Honneth 1992). Hier setzt die intersektionale Analyse $^{7}$ als weiterer Hauptbestandteil des Lehrer- und

7 „Intersektionalität“ wird hier weniger als Forschungstheorie denn als theoretische Konzeption mit normativem Anspruch begriffen (Crenshaw 2013.) 
Lehrerinnenhabitus an, indem soziale Differenzlinien bzw. potenzielle Diskriminierungskategorien mit Blick auf die Verwirklichung eines humanisierenden, selbstbefähigenden, demokratischen und egalitären Toleranz- bzw. Bildungsbegriffes kritisch reflektiert werden - beispielsweise im Sinne Herbert Marcuses (1970): Toleranz braucht die Parteinahme für das „Gute“ und die Humanität in der Lehramtsausbildung. Auf diesem Weg können Potenziale und vorliegende Barrieren in Lern-Lehrsituationen adäquater eingeschätzt werden, die ihrerseits Positiv- bzw. Negativspiralen im Hinblick auf individuelle wie soziale Lernprozesse auslösen können. Ein solchermaßen macht- und herrschaftskritischer, reflexiver Lehrer- und Lehrerinnenhabitus stellt zudem innovative Optionen bereit, bildungswissenschaftliches Diskutieren und didaktisches Handeln in Bezug auf Inklusive Migrationspädagogik voranzutreiben, um ein systematischeres Verständnis inklusiv-migrationspädagogischer Professionalität im Zusammenhang mit einem reflexiven Lehrer- und Lehrerinnenhabitus zu ermöglichen.

\section{Ausblick}

Fragen nach gesellschaftlicher Verantwortung und dem kritischen Potenzial von Bildung und Erziehung, das sich in Bildungsinstitutionen realisiert, werden dieser Tage angesichts populistischer Welt- und Menschenbilder zunehmend in den Hintergrund gedrängt. Universitäre Studien stehen immer mehr unter dem neoliberalen Erwartungsdruck, ökonomisch „,anwendbar“ und „unmittelbar" verfügbar zu sein. Eine sich selbst in dieser Weise entfremdete Bildung ist prinzipiell entpolitisiert: Was nur zählt, sind "messbare Daten“, während Fragen nach Werten und Normen, sozialer Gerechtigkeit, gesellschaftlicher Verantwortung und Humanität tendenziell als „nichtwissenschaftliche Angelegenheiten“" aus dem Blick geraten oder gar von vornherein „ausgeschieden“ werden. Dagegen steht der kritisch-theoretische Anspruch, dass Bildungsinstitutionen wie Schulen, Hochschulen, Akademien und Universitäten nicht außerhalb von Gesellschaft, sozialen Strukturen und Diskursen stehen. Demnach können sie auch nicht als ein apolitisches „Feld“ oder „apolitischer Lern- und Bildungs-Raum“ betrachtet werden.

In der „PädagogInnenbildung Neu“ in Österreich wird von einem „reflexiven Lehrer- und Lehrerinnenhabitus" gesprochen, ohne diesen Begriff näher zu bestimmen. Dem auf Grundlage der zentralen philosophischen Konzepte Horkheimers bzw. Adornos ver- tiefend nachzugehen, um theoretisch zu reflektieren, was unter einer „kritischen“ und „reflexiven“ Haltung bei Lehrern und Lehrerinnen überhaupt verstanden werden bzw. wie Lehrer und Lehrerinnen mit ihrer Rolle als „ideologischen Multiplikatoren“ umgehen könnte(n), ist notwendig. Nur so kann die österreichische Lehramtsausbildung, die sich die Vermittlung eines „kritischen Lehrer- und Lehrerinnenhabitus“ zum Ziel setzt, auch hinsichtlich ihrer (impliziten oder expliziten) „politischen Funktionen“ kritisch reflektiert werden. Dieses kritische Denken kann gerade darauf beruhen, die von den Bildungsinstitutionen selbst vertretenen Werte mit ihrer gesellschaftlichen Wirklichkeit zu konfrontieren und damit nicht zuletzt auf deren Einlösung zu pochen.

Dieses „Eingedenken“ der Natur im Subjekt besagt dann weniger, eine ursprüngliche, nicht entfremdete Einheit von Mensch und Natur zu unterstellen, vielmehr enthüllt die Natur gerade unter der Perspektive des voll entfalteten menschlichen Machbarkeitsstrebens ihre Gleichgültigkeit gegenüber dem Schicksal des Einzelwesens. Dieses „Eingedenken“ kann Gewalt und Leiden zwar nicht rückgängig machen, sondern nur erinnernd aufbewahren, aber damit hilft es nichtsdestoweniger dabei, die instrumentalistische Verhärtung des Subjekts in seinem Umgang mit seiner inneren und äußeren Natur und mit anderen Subjekten zu mildern (Böhme 2004: 106). Die Entwicklung der Menschheitsgeschichte ist für die kritische Theorie daher so lange naturwüchsig, blind und fremdbestimmt, wie der Mensch das Bewusstsein seiner eigenen Naturhaftigkeit, seiner eigenen Teilhabe am Naturzusammenhang verdrängt.

Auch im Rahmen der österreichischen Lehramtsausbildung kann die Kluft zwischen bloßer empirischer Bildungstatsachenforschung und Sozialphilosophie überwunden werden: „In dieser Funktion wäre Philosophie das Eingedenken und Gewissen der Menschheit und hülfe dadurch, den Weg der Menschheit davor zu bewahren, der sinnlosen Runde des Anstaltsinsassen während seiner Erholungsstunde ähnlich zu werden“ (Horkheimer 1992b: 173). „Im Eingedenken der Natur im Subjekt“ der Lehramtsausbildung bzw. im individuellen Bewusstsein des Lehrers bzw. der Lehrerin ist Versöhnung durch Reflexion aus Perspektive der Aufklärung sehr wohl möglich - eine Versöhnung seiner bzw. ihrer äußeren und inneren Natur mit seinem bzw. ihrem Vernunftdenken und damit die Einsicht in die Entzweiung seiner bzw. ihrer Natur und Vernunft. Um es mit Böhme zu sagen: „Es ist das Subjekt, das erkennt, 
dass es nicht Herr im eigenen Haus ist, das Subjekt, zu dem das Erleiden ebenso gehört wie das Handeln. Es ist der Mensch, dem Eingedenken im Subjekt nicht bloß Reflexion bleibt, sondern zur alltäglich geübten Praxis wird“ (Böhme 2004: 107). Mit Blick auf den aktuell weltweit $\mathrm{zu}$ beobachtenden Trend hin $\mathrm{zu}$ populistischen Strömungen kann der pädagogische Imperativ einer „Erziehung nach Ausschwitz“ (Adorno) bzw. das Konzept einer kritischen Lehrendenpersönlichkeit im Sinne der kritischen Theorie dem aktuellen Schul- und Hochschulweisen aus dieser Perspektive sehr wohl beistehen.

Kritisches Sinnieren und grundlagenorientiertes Theoretisieren müssen außerdem nicht unmittelbar in praktisch-pädagogische Lösungsvorschläge münden. Es braucht die intensive Auseinandersetzung mit grundlagenorientierten theoretischen Texten in der Lehrendenausbildung, um politisch denken zu können. Es geht um ein politisches Bildungswissen, das ein kritisches Bewusstsein für die Lehrendenausbildung erst ermöglicht, um sich selbst der politischen Dimension des eigenen Handelns als Lehrende oder Lehrender im gesamtgesellschaftlichen Kontext gewahr zu werden, um politisch gebildet zu sein und politisch (aus-)bilden zu können. Ein damit einhergehender Bildungsauftrag an Pädagogischen Hochschulen setzt bei den Eigenschaften der Lehrenden, den Merkmalen der Bildungsinstitutionen, den dort praktizierten Lehrformen und Lehrinhalten und den Haltungen der Studierenden an. Der Anspruch der kritischen Theorie als kritische Gesellschaftstheorie steigt und fällt mit der erworbenen theoretisch-reflexiven Kompetenz von Lehrenden gemäß den Curricula der Lehramtsausbildung.

\section{Literatur}

Adorno, T.W. (2014): Vorlesung über Negative Dialektik. Fragmente zur Vorlesung 1965/66. Frankfurt/Main: Suhrkamp.

Adorno, T.W. (2013): Erziehung zur Mündigkeit. Vorträge und Gespräche. Berlin: Suhrkamp.

Adorno, T.W. (200o): Negative Dialektik. Frankfurt/Main: Suhrkamp.

Adorno, T.W. (1998): Theorie der Halbbildung. In: Tiedemann, R. (Hg.): Gesammelte Schriften Band 8. Soziologische Schriften I. Darmstadt: Wissenschaftliche Buchgesellschaft: 93-121.

Adorno, T.W. (1997a): Erziehung nach Auschwitz. In: Tiedemann, R. (Hg.): Gesammelte Schriften Band 10/2. Kul- turkritik und Gesellschaft. Frankfurt/Main: Suhrkamp: 674-69o.

Adorno, T.W. (1997b): Tabus über den Lehrberuf. In: Tiedemann, R. (Hg.): Gesammelte Schriften Band 10/2. Kulturkritik und Gesellschaft. Frankfurt/Main: Suhrkamp: 656-673.

Armin, B./Rothermel, L. (2001) (Hg.): Handbuch kritische Pädagogik: eine Einführung in die Erziehungs- und Bildungswissenschaft. Weinheim u.a.: Deutscher Studienverlag.

Baumert, J. (2007): Was messen internationale Schulleistungsstudien? Resultate kumulativer Wissenserwerbsprozesse. Eine Antwort auf Heiner Rindermann. Psychologische Rundschau, 58 (2), 118-145.

Baumert, J./Tillmann, K.-J. (2016) (Hg.): Empirische Bildungsforschung: Der kritische Blick und die Antwort auf die Kritiker. Zeitschrift für Erziehungswissenschaft, Sonderheft, 19 (31).

Bellmann, J./Müller, T. (2011) (Hg.): Wissen, was wirkt: Kritik evidenzbasierter Pädagogik. Wiesbaden: VS.

Bernhard, A. (2001): Bildung und Erziehung: Grundlagen emanzipativer Subjektwerdung: Beiträge zur kritischen Bildungstheorie und Pädagogik. Kiel: Götzelmann.

Booth, T./Ainscow, M. (2011): Index of Inclusion. Developing Learning and Participation in Schools. Bristol: CSIE.

Bourdieu, P. (1995): Narzisstische Reflexivität und wissenschaftliche Reflexivität. In: Berg, E./Fuchs, M. (Hg.): Kultur, soziale Praxis, Text. Die Krise der ethnographischen Repräsentation. Frankfurt/Main: Suhrkamp: 365-374.

Bourdieu, P. (1983): Ökonomisches Kapital, kulturelles Kapital, soziales Kapital. In: Kreckel, R. (Hg.): Soziale Ungleichheiten. Göttingen: Otto Schwartz \& Co: 183-198.

Bourdieu, P./Passeron. J.-C. (1971): Die Illusion der Chancengleichheit: Untersuchungen zur Soziologie des Bildungswesens am Beispiel Frankreichs. Stuttgart: Klett.

Böhme, G. (2004): Eingedenken der Natur im Subjekt oder: die Geburt des Subjekts aus dem Schmerz. In: Gruschka, A./Oevermann, U. (Hg.): Die Lebendigkeit der kritischen Gesellschaftstheorie: Dokumentation der Arbeitstagung aus Anlass des 100. Geburtstages von Theodor W. Adorno; 4.-6. Juli 2003 an der Johann Wolfgang GoetheUniversität, Frankfurt/Main. Wetzlar: Büchse d. Pandora: 97-108.

Bramberger, A./Kronberger, S./Oberlechner, M. (2017) (Hg.): Bildung - Intersektionalität - Geschlecht. Innsbruck, Wien, Bozen: Studienverlag

Brügelmann, H. (2015): Vermessene Pädagogik - Standardisierte Schüler. Weinheim: Beltz.

Claußen, B./Scarbath, H. (1979) (Hg.): Konzepte einer kritischen Erziehungswissenschaft. München u.a.: Reinhardt.

Crenshaw, K. (2013): „Die Intersektion von „Rasse“ und Geschlecht demarginalisieren: Eine Schwarze feministische Kritik am Antidiskriminierungsrecht, der feministischen Theorie und antirassistischen Politik“. In: Lutz, H./ Herrera Vivar, M./Supik, L. (Hg.): Fokus Intersektiona- 
lität: Bewegungen und Verortungen eines vielschichtigen Konzeptes. Wiesbaden: VS: 35-58.

Doğmuş, A./Karakaşoğlu, Y./Mecheril, P. (2016): Pädagogisches Können in der Migrationsgesellschaft. Wiesbaden: VS.

Fend, H. (1980): Theorie der Schule. München u.a.: Urban \& Schwarzenberg.

Friebertshäuser, B./Rieger-Ladich, M./Wigger, L. (2009) (Hg.): Reflexive Erziehungswissenschaft: Forschungsperspektiven im Anschluss an Pierre Bourdieu. Wiesbaden: VS.

Gruschka, A. (2006): Bildungsstandards oder das Versprechen, Bildungstheorie in empirischer Bildungsforschung aufzuheben. Pädagogische Korrespondenz, 19 (35), 5-22.

Gruschka, A. (2004): Kritische Pädagogik nach Adorno. In: Gruschka, A./Oevermann, U. (Hg.): Die Lebendigkeit der kritischen Gesellschaftstheorie: Dokumentation der Arbeitstagung aus Anlass des 100. Geburtstages von Theodor W. Adorno; 4.-6. Juli 2003 an der Johann Wolfgang Goethe-Universität, Frankfurt/Main. Wetzlar: Büchse d. Pandora: 135-160.

Gruschka, A. (2003): Vortrag: „Die Lebendigkeit kritischer Gesellschaftstheorie" im Rahmen einer Arbeitstagung aus Anlass des 10o. Geburtstages von Theodor W. Adorno am 4.-6. Juli 2003 an der Johann Wolfgang Goethe-Universität, Frankfurt/Main. Online: http://publikationen. ub.uni-frankfurt.de/frontdoor/index/index/docId/4557 [2.1.2018].

Gruschka, A./Oevermann, U. (2004) (Hg.): Die Lebendigkeit der kritischen Gesellschaftstheorie: Dokumentation der Arbeitstagung aus Anlass des 100. Geburtstages von Theodor W. Adorno; 4.-6. Juli 2003 an der Johann Wolfgang Goethe-Universität, Frankfurt/Main. Wetzlar: Büchse d. Pandora.

Häcker, T. (2017): Grundlagen und Implikationen der Forderung nach Förderung von Reflexivität in der Lehrerinnen- und Lehrerbildung. In: Berndt, C./Häcker, T./ Leonhard, T. (Hg.): Reflexive Lehrerbildung revisited: Traditionen - Zugänge - Perspektiven. Bad Heilbronn: Klinkhardt: 21-45.

Helsper, W. (2001): Praxis und Reflexion. Die Notwendigkeit einer „doppelten Professionalisierung“ des Lehrers. Journal für Lehrerinnen- und Lehrerbildung, 1 (3), 7-15.

Herzog, W. (2016): Kritik der evidenzbasierten Pädagogik. In: Baumert, J./Tillmann, K.-J. (Hg.): Empirische Bildungsforschung: Der kritische Blick und die Antwort auf die Kritiker. Zeitschrift für Erziehungswissenschaft, Sonderheft, 19 (31), 201-213.

Honneth, A. (1992): Kampf um Anerkennung: Zur moralischen Grammatik sozialer Konflikte. Frankfurt am Main: Suhrkamp.

Horkheimer, M. (1992a): Die Revolte der Natur. In: Schmidt, A. (Hg.): Zur Kritik der instrumentellen Vernunft: Aus den Vorträgen und Aufzeichnungen seit Kriegsende. Frankfurt/Main: Fischer: 93-123.

Horkheimer, M. (1992b): Zum Begriff der Philosophie. In: Schmidt, A. (Hg.): Zur Kritik der instrumentellen Ver- nunft: Aus den Vorträgen und Aufzeichnungen seit Kriegsende. Frankfurt/Main: Fischer, 153-174.

Horkheimer, M. (1992c): Mittel und Zwecke. In: Schmidt, A. (Hg.): Zur Kritik der instrumentellen Vernunft: Aus den Vorträgen und Aufzeichnungen seit Kriegsende. Frankfurt/Main: Fischer: 15-62.

Horkheimer, M. (1989): Kritik des Positivismus. In: Schmidt, A./ Schmid Noerr, G. (Hg.): Gesammelte Schriften Frankfurt/Main: Fischer: 347-396.

Horkheimer, M. (1988): Die gesellschaftliche Funktion der Philosophie. In: Schmidt, A./Schmid Noerr, G. (Hg.): Gesammelte Schriften. Frankfurt/Main: Fischer: 332-351.

Horkheimer, M. (1985a): Begriff der Bildung. In: Schmidt, A./ Schmid Noerr, G. (Hg.): Gesammelte Schriften: Vorträge und Aufzeichnungen 1949-1973. Frankfurt/Main: Fischer: 409-419.

Horkheimer, M. (1985b): Verantwortung und Studium. In: Schmidt, A./Schmid Noerr, G. (Hg.): Gesammelte Schriften: Vorträge und Aufzeichnungen 1949-1973. Frankfurt/ Main: Fischer: 433-453.

Horkheimer, M. (1985c): Akademische Freiheit. In: Schmidt, A./ Schmid Noerr, G. (Hg.): Gesammelte Schriften: Vorträge und Aufzeichnungen 1949-1973. Frankfurt/Main: Fischer: 420-432.

Horkheimer, M. (1985d): „Zum Tode Adornos“. In: Schmidt, A./Schmid Noerr, G. (Hg.): Gesammelte Schriften. Frankfurt/Main: Fischer: 284-288.

Horkheimer, M. (1970): Traditionelle und kritische Theorie: Vier Aufsätze. Frankfurt/Main: Fischer.

Horkheimer, M./Adorno, T.W. (1987): Dialektik der Aufklärung. In: Schmidt, A./Schmid Noerr, G. (Hg.): Gesammelte Schriften. „Dialektik der Aufklärung“ und Schriften 1940-1950. Frankfurt/Main: Fischer: 11-290.

Jornitz, S. (2009): Evidenzbasierte Bildungsforschung. Pädagogische Korrespondenz, 40, 68-75.

Kronberger, S./Kühberger, C./Oberlechner, M. (2016) (Hg.): Diversitätskategorien in der Lehramtsausbildung: Ein Handbuch. Innsbruck, Wien, Bozen: Studienverlag.

Lenzen, D. (1991): Pädagogisches Risikowissen, Mythologie der Erziehung und pädagogische Méthexis. Auf dem Weg zu einer reflexiven Erziehungswissenschaft. In: Oelkers, J./Tenorth, H.-E. (Hg.): Pädagogisches Wissen. Zeitschrift für Pädagogik: 27. Beiheft. Weinheim/Basel: Beltz, 109-125.

Marcuse, H. (1970): Repressive Toleranz. In: Wolff, R.P./Moore, B. (Hg.): Herbert Marcuse. Kritik der reinen Toleranz. Frankfurt/Main: Suhrkamp: 91-128.

Mecheril, P. (2004): Einführung in die Migrationspädagogik. Weinheim u.a.: Beltz.

Mecheril, P./Oberlechner, M. (2016): Migration bildet. Anforderungen an pädagogisches Handeln. In: Kronberger, S./Kühberger, C./Oberlechner, M. (Hg.): Diversitätskategorien in der Lehramtsausbildung: Ein Handbuch. Innsbruck, Wien, Bozen: Studienverlag: 153-166.

Oberlechner, M. (2017a): Migration Pedagogy as an Inclusive Educational Enterprise for Austrian Schools and Uni- 
versities of Pedagogy. In: Oberlechner, M./TrültzschWijnen, C./Duval, P. (Hg.): Migration bildet. Migration Educates. Baden-Baden: Nomos: 35-68.

Oberlechner, M. (2017b): Aspekte einer soziologischen Migrationspädagogik: multikausal, plurifaktoriell, intersektional. In: Bramberger, A./Kronberger, S./Oberlechner, M. (Hg.): Bildung - Intersektionalität - Geschlecht. Innsbruck, Wien, Bozen: Studienverlag: 83-121.

Oberlechner, M./Trültzsch-Wijnen, C./Duval, P. (2017) (Hg.): Migration bildet. Migration Educates. Baden-Baden: Nomos

Prengel, A. (2006): Pädagogik der Vielfalt: Verschiedenheit und Gleichberechtigung in Interkultureller, Feministischer und Integrativer Pädagogik. Wiesbaden: VS.

Radtke, F.O. (2015): Methodologischer Ökonomismus. Organische Experten im Erziehungssystem. Erziehungswissenschaft, 26 (50), 7-16.

Reich, K. (2012) (Hg.): Inklusion und Bildungsgerechtigkeit: Standards und Regeln zur Umsetzung einer inklusiven Schule. Weinheim: Beltz.

Schön, D. A. (1983): The Reflective Practitioner. How Professionals Think in Action. New York: Basic Books.

Tenorth, H.-E. (2014): Evidenzbasierte Bildungsforschung vs. Pädagogik als Kulturwissenschaft - Über einen neuerlichen Paradigmenstreit in der wissenschaftlichen Pädagogik. Online: http://nevelestudomany.elte. hu/downloads/2014/nevelestudomany_2014_3_5-21.pdf [20.7.2018].

Terhart, E. (2002): Wie können die Ergebnisse von vergleichenden Leistungsstudien systematisch zur Qualitätsverbesserung in Schulen genutzt werden? Zeitschrift für Pädagogik, 48 (1), 91-110.

UNESCO (1994): The Salamanca Statement and Framework for Action on Special Needs Education. Paris. Online: http://bidok.uibk.ac.at/library/unesco-salamanca.html [2.1.2018].

Williamon, A. (2009) (Hg.): Proceedings of the International Symposium on Performance Science 2009. Utrecht: Assoc. Européenne des Conservatoires, Acad. de Musique et Musikhochschulen (AEC).

Zinnecker, J. (1975) (Hg.): Der heimliche Lehrplan: Untersuchungen zum Schulunterricht. Weinheim u.a.: Beltz. 